KYUNGPOOK Math. J. 53(2013), 407-417

http://dx.doi.org/10.5666/KMJ.2013.53.3.407

\title{
t-Prüfer Modules
}

MyeOng Og KIM

Department of Mathematics, Kyungpook National University, Taegu 702-701, Ko-

rea

e-mail : myngokim@knu.ac.kr

HWANKOO KIM*广

Department of Information Security, Hoseo University, Asan 336-795, Korea

e-mail : hkkim@hoseo.edu

Dong YeOL OH

Division of Liberal Arts, Hanbat National University, Daejeon 305-719, Korea

e-mail : dongyeol70@gmail.com

ABstRACT. In this article, we characterize $t$-Prüfer modules in the class of faithful multiplication modules. As a corollary, we also characterize Krull modules. Several properties of a $t$-invertible submodule of a faithful multiplication module are given.

\section{Introduction}

Let $R$ be a commutative ring with identity and $M$ be a unital $R$-module. $M$ is said to be faithful if $\operatorname{ann}_{R}(M)=0$. $M$ is called a multiplication module if each submodule $N$ of $M$ has the form $I M$ for some ideal $I$ of $R$, equivalently, for any submodule $N$ of $M, N=\left(N:_{R} M\right) M . M$ is called a cancellation module if for all ideals $I$ and $J$ of $R, I M \subseteq J M$ implies $I \subseteq J$. It was shown in [5, Proposition 2.2 ] that if $R$ is an integral domain and $M$ is a faithful multiplication $R$-module, then $M$ is finitely generated. Thus it follows from [12, Theorem 3.1] that a faithful multiplication module $M$ over an integral domain is a cancellation module. Hence we have that $I\left(N:_{R} M\right)=\left(I N:_{R} M\right)$ for all submodules $N$ of $M$ and all ideals $I$ of $R$. It was also shown in [13, Lemma 2.1] that if $M$ is a faithful multiplication $R$-module over integral domain $R$, then $M$ is torsion-free.

* Corresponding Author.

$\dagger$ This research was supported by Basic Science Research Program through the National Research Foundation of Korea(NRF) funded by the Ministry of Education, Science and Technology(2010-0011996).

Received December 8, 2012; accepted April 19, 2013.

2010 Mathematics Subject Classification: 13A15, 13F05, 13C13.

Key words and phrases: $t$-Prufer module, faithful multiplication module, Krull module, $t$-invertible submodule. 
Let $R$ be an integral domain with quotient field $K$ and $M$ be a unital $R$-module. Let $N$ be a nonzero submodule of $M$ and let $N^{-1}=\left(M:_{K} N\right)=\{x \in K \mid x N \subseteq$ $M$ \}. Then $N^{-1}$ is an $R$-submodule of $K$ and $N N^{-1} \subseteq M$. Following Naoum and Al-Alwan, in [1], $N$ is said to be invertible in $M$ if $N N^{-1}=M$. Clearly $M$ is invertible in $M$, and it is proved in [1, Remark 3.8] that $R$ is an integral domain if and only if every nonzero cyclic submodule of the faithful multiplication $R$-module $M$ is invertible in $M$ (i.e., $M$ is a $D_{1}$-module).

Let $M$ be a faithful multiplication module over an integral domain $R$ and let $N$ be a submodules of $M$. In [4], M. Ali defined $N_{v}=\left(N^{-1}\right)^{-1}=R:_{K}\left(M:_{K} N\right)$ and showed that $N^{-1}=\left(N:_{R} M\right)^{-1}$, and hence $N_{v}=\left(N:_{R} M\right)_{v}$ (in particular, $M_{v}=R$ ), and then introduced the concept of a divisorial submodule or $v$-submodule of $M$ as follows: $N$ is a $v$-submodule if $N=N_{v} M . N$ is called a $v$-submodule of finite type if $N=L_{v} M$ for some finitely generated submodule $L$ of $M$. It follows that $N$ is a $v$-submodule of $M$ if and only if $\left(N:_{R} M\right)$ is a $v$-ideal of $R$. If $L$ and $N$ are submodules of a multiplication module $M$ with $L \subseteq N$, then $L_{v} \subseteq N_{v}$. It was also shown that for any submodule $N$ of a multiplication $R$-module, $N \subseteq N_{v} M$. If $N$ is a submodule of $M$ and $I$ is an ideal of $R$ such that either $I_{v}$ is invertible or $N_{v}$ is invertible, then as it was remarked in $\left[4\right.$, p.144] it is easily seen that $(I N)_{v}=\left(I N:_{R}\right.$ $M)_{v}=\left(I\left(N:_{R} M\right)\right)_{v}=I_{v}\left(N:_{R} M\right)_{v}=I_{v} N_{v}$. In [16], we introduced the concept of $t$-invertible submodule of a multiplication module and gave some characterizations of faithful multiplication Krull modules, Mori modules and $\pi$-modules. Also, we defined $N_{t}=\bigcup\left\{L_{v} \mid L\right.$ is a finitely generated submodule of $M$ contained in $\left.N\right\}$. It is easily seen that $N_{t}$ is an $R$-submodule of $K, N_{t} \subseteq N_{v}$ and $\left(N N^{-1}\right)_{t} \subseteq R$. $N$ is said to be $t$-(resp. $v$-)invertible submodule of $M$ if $\left(N N^{-1}\right)_{t}=R$ (resp. $\left(N N^{-1}\right)_{v}=R$ ). It is clear that every invertible submodule of $M$ is $t$-invertible and every $t$-invertible submodule is $v$-invertible. By [6, Proposition 2.2], $N$ is finitely generated if and only if $\left(N:_{R} M\right)$ is finitely generated. Using this result, we have that $\left(N:_{R} M\right)_{t}=N_{t}$ and $N \subseteq N_{t} M$. So we call $N$ a $t$-submodule of $M$ if $N=N_{t} M . N$ is called a t-submodule of finite type if $N=L_{t} M$ for some finitely generated submodule $L$ of $M$. Clearly every $v$-submodule is a $t$-submodule. If $I$ is a $t$-ideal of $R$, then $I M$ is a $t$-submodule of $M$. Also if $N$ is finitely generated, then $N$ is a $v$-submodule of $M$ if and only if $N$ is a $t$-submodule of $M$.

In section 2 we characterize faithful multiplication $t$-Prüfer modules (i.e., every nonzero finitely generated submodule is $t$-invertible). In section 3 we give several properties of a $t$-invertible submodule of a faithful multiplication module.

\section{2. $t$-Prüfer Modules}

We begin with this section by giving a characterization of when a submodule of the module is $*$-submodule of finite type.

Lemma 2.1. Let $M$ be a faithful multiplication module over an integral domain $R$, $I$ an ideal of $R, N$ a submodules of $M$, and let $*=v$ or $t$. Then

(1) $N$ is a $*$-submodule of finite type if and only if $\left(N:_{R} M\right)$ is a $*$-ideal of finite 
type.

(2) $I$ is a $*$-ideal of finite type if and only if IM is a *-submodule of finite type.

Proof. Since the assertion for the case $*=v$ is similar to that of $*=t$, we only consider the case $*=t$. (1) If $N$ is a $t$-submodule of finite type, then $N=L_{t} M$ for some finitely generated submodule $L$ of $M$ and $\left(N:_{R} M\right)$ is a $t$-ideal by [16, Proposition 2.2]. Since $N_{t}=\left(N:_{R} M\right)_{t}$, we have that $\left(N:_{R} M\right)_{t} M=\left(L:_{R} M\right)_{t} M$.

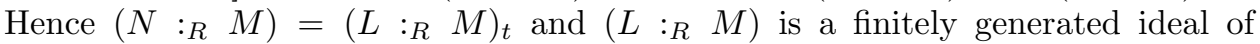
$R$. Conversely, assume that $\left(N:_{R} M\right)$ is a $t$-ideal of finite type. Then by $[16$, Proposition 2.2], $\left(N:_{R} M\right)=I_{t}$ for some finitely generated ideal $I$ of $R$ and $N$ is a $t$-submodule. Hence $N=\left(N:_{R} M\right) M=I_{t} M=\left(I M:_{R} M\right)_{t} M=(I M)_{t} M$ and $I M$ is a finitely generated submodule of $M$.

Proposition 2.2. The following conditions are equivalent for a faithful multiplication module $M$ over an integral domain $R$.

(1) Each $t$-submodule of $M$ is of finite type.

(2) $M$ satisfies the ascending chain condition on t-submodules.

(3) $M$ satisfies the ascending chain condition on v-submodules.

(4) For each nonzero submodule $N$ of $M$, there exists a finitely generated submodule $L$ of $M$ contained in $N$ such that $N_{t}=L_{t}$.

Proof. The equivalences of (1), (2) and (3) follow from Lemma 2.1 and [20, Theorem 1.1]. To complete the proof, we show that $(1) \Leftrightarrow(4)$.

$(1) \Rightarrow(4)$. By Lemma 2.1, each $t$-ideal of $R$ is of finite type. Then for each nonzero ideal $I$, there exists a finitely generated ideal $J$ of $R$ contained in $I$ such that $I_{t}=J_{t}[20$, Theorem 1.1]. Let $N$ be a nonzero submodule of $M$. Since $M$ is a multiplication module, $\left(N:_{R} M\right)$ is a nonzero ideal of $R$. Then $\left(N:_{R} M\right)_{t}=B_{t}$ for some finitely generated ideal $B \subseteq\left(N:_{R} M\right)$ of $R$. Thus $(B M)_{t}=\left(B M:_{R} M\right)_{t}=$ $B_{t}=N_{t}$ and $B M \subseteq N$ is a finitely generated submodule of $M$.

(4) $\Rightarrow(1)$. Let $N$ be any $t$-submodule of $M$. Then by hypothesis, there exists a finitely generated submodule $L$ of $M$ contained in $N$ such that $N_{t}=L_{t}$. Thus $N=N_{t} M=L_{t} M$, and so $N$ is of finite type.

Recall from [3, p. 25] that an $R$-module $M$ is called a cyclic submodule module (CSM) if every submodule of $M$ is cyclic. It was shown in [3] that a faithful multiplication module over an integral domain $R$ is a CSM if and only if $R$ is a principal ideal domain. In [16], we call an $R$-module $M$ a $v$-Prüfer module if $\left(N N^{-1}\right)_{v}=R$ for every nonzero finitely generated submodule $N$ of $M$ and $M$ a Krull module if $M_{P}$ is a CSM $R_{P}$-module for each $P \in X^{(1)}(R)$, which is the set of height-one prime ideals of $R, M=\bigcap_{P \in X^{(1)}(R)} M_{P}$, and each nonzero $x \in M$ is primitive in all but a finite number of $M_{P}$. It was shown in [16, Theorem 2.1(1) and Theorem 2.8] that a faithful multiplication module over an integral domain $R$ is a 
$v$-Prüfer (resp., Krull) module if and only if $R$ is a $v$-Prüfer (resp., Krull) domain. The following result is an analogue to Cohen's theorem for $v$-Prüfer modules.

Theorem 2.3. Let $R$ be an integral domain and $M$ a faithful multiplication and $v$-Prüfer module. If every prime $t$-submodule of $M$ is of finite type, then every $t$-submodule of $M$ is of finite type.

Proof. By [16, Theorem 2.5], $M$ is a $v$-Prüfer module if and only if $R$ is a $v$-Prüfer domain. Let $P$ be any prime $t$-ideal of $R$. Then $P M$ is a prime $t$-submodule of $M$. Since $P M$ is a $t$-submodule of finite type, we have $P M=Q_{t} M$ for some finitely generated submodule $Q$ of $M$. Then $P=\left(Q:_{R} M\right)_{t}$ and $\left(Q:_{R} M\right)$ is a finitely generated ideal of $R$. Hence $P$ is a $t$-ideal of finite type. By [17, Theorem 2.3], every $t$-ideal of $R$ is of finite type. Let $N$ be a $t$-submodule of $M$. Then $\left(N:_{R} M\right)$ is a $t$-ideal of finite type, and so $\left(N:_{R} M\right)=B_{t}$ for some finitely generated ideal $B$ of $R$. Hence $N=\left(N:_{R} M\right) M=B_{t} M=\left(B M:_{R} M\right)_{t} M=(B M)_{t} M$ and $B M$ is a finitely generated submodule of $M$.

Several characterizations of Krull modules were given in [16]. In the following, we characterize Krull modules in terms of $v$-Prüfer modules, which generalizes [17, Theorem 2.4].

Corollary 2.4. Let $R$ be an integral domain and $M$ a faithful multiplication and $v$-Prüfer module. Then the following are equivalent.

(1) The ascending chain condition on t-submodules holds.

(2) Each t-submodule of $M$ is of finite type (and hence each $v$-submodule of $M$ is of finite type)

(3) Each prime $t$-submodule is of finite type.

(4) $M$ is a Krull module.

Proof. Note first that $R$ is a $v$-Prüfer domain ([16, Theorem 2.5(1)]).

(1) $\Leftrightarrow(2)$ This follows from Lemma 2.1 and [17, Theorem 2.4].

$(2) \Leftrightarrow(3)$ This follows from Lemma 2.1, [17, Theorem 2.4] and Theorem 2.3.

$(2) \Rightarrow(4)$ Let $I$ be any $t$-ideal of $R$. Since $I M$ is a $t$-submodule, $I M$ is of finite type. Then $I$ is of finite type by Lemma 2.1 . Thus by again [17, Theorem 2.4] $R$ is a Krull domain. It follows from [16, Theorem 2.8] that $M$ is a Krull module.

$(4) \Rightarrow(2)$ By [16, Theorem 2.8], $R$ is a Krull domain. Then every $t$-ideal of $R$ is of finite type. Let $N$ be a $t$-submodule of $M$. Then $\left(N:_{R} M\right)$ is a $t$-ideal of finite type by Lemma 2.1. Thus $N$ is of finite type.

We recall that an integral domain $R$ is a $t$-Prüfer domain (or Prüfer $v$ multiplication domain (for short $P v M D)$ ) if $\left(I I^{-1}\right)_{t}=R$ for every nonzero finitely generated ideal $I$ of $R$. Now we extend this concept to the module case: An $R$ module $M$ is a $t$-Prüfer module if $\left(N N^{-1}\right)_{t}=R$ for every nonzero finitely generated submodule $N$ of $M$. 
Lemma 2.5. Let $M$ be a faithful multiplication module over an integral domain $R$. Then $M$ is a t-Prüfer module if and only if $R$ is a $t$-Prüfer domain.

Proof. Let $I$ be a nonzero finitely generated ideal of $R$. Since $M$ is a faithful multiplication module, $I M$ is a nonzero finitely generated submodule of $M$. So $I M$ is $t$-invertible. By [16, Lemma 2.1], $I$ is $t$-invertible. Thus $R$ is a $t$-Prüfer domain. Conversely, let $N$ be a nonzero finitely generated submodule of $M$. Then $\left(N:_{R} M\right)$ is a nonzero finitely generated ideal of $R$, and so $\left(N:_{R} M\right)$ is $t$-invertible. Thus $N$ is $t$-invertible by [16, Proposition 2.2]. Hence $M$ is a $t$-Prüfer module.

Recall from [3] that an $R$-module $M$ is called a valuation module if for all $m, n \in M$, either $R m \subseteq R n$ or $R n \subseteq R m$, or equivalently, for all submodules $L, N$ of $M$, either $L \subseteq N$ or $N \subseteq L$. It was shown that a faithful multiplication module over an integral domain $R$ is a valuation module if and only if $R$ is a valuation domain.

Theorem 2.6. Let $M$ be a faithful multiplication module over an integral domain $R$. Then the following are equivalent.

(1) $M$ is a t-Prüfer module.

(2) For every maximal t-ideal $P$ of $R, M_{P}$ is a valuation module.

(3) Every v-submodule of finite type is t-invertible.

Proof. (1) $\Leftrightarrow(2)$ If $M$ is a $t$-Prüfer module, then $R$ is a $t$-Prüfer domain by Lemma 2.5. Then $R_{P}$ is a valuation domain for every maximal $t$-ideal $P$ of $R$. Since $M_{P}$ is a faithful multiplication $R_{P}$-module, $M_{P}$ is a valuation module by [5, Proposition 2.2]. Conversely, if $M_{P}$ is a valuation module, then by [5, Proposition 2.2] $R_{P}$ is a valuation domain. Thus $R$ is a $t$-Prüfer domain. Hence $M$ is a $t$-Prüfer module by Lemma 2.5.

$(1) \Leftrightarrow(3)$ Suppose that $M$ is a $t$-Prüfer module and let $N$ be a $v$-submodule of finite type. By Lemma $2.1,\left(N:_{R} M\right)$ is a $v$-ideal of finite type. Then $\left(N:_{R}\right.$ $M)=B_{v}$ for some finitely generated ideal $B \subseteq\left(N:_{R} M\right)$. Since $B M$ is finitely generated, we have $B M$ is $t$-invertible, and so $B$ is $t$-invertible by [16, Lemma 2.1]. Then $\left(N:_{R} M\right)$ is $t$-invertible. By [16, Proposition 2.2], $N$ is $t$-invertible. Conversely, let $N$ be a nonzero finitely generated submodule of $M$. Since $M$ is a faithful multiplication module, $\left(N:_{R} M\right)$ is finitely generated. Then $I=\left(N:_{R} M\right)_{v}$ is a $v$-ideal of finite type. By Lemma 2.1, $I M$ is a $v$-submodule of finite type. So $I M$ is $t$-invertible. Then $\left(N:_{R} M\right)$ is $t$-invertible, and so $N$ is $t$-invertible. Hence $M$ is a $t$-Prüfer module.

Let $M$ be a faithful multiplication module over an integral domain $R$. By [16, Lemma 2.1 and Proposition 2.2], every nonzero ideal of $R$ is $v$-invertible if and only if every nonzero submodule of $M$ is $v$-invertible. Recall from [16] that $M$ is called an essential module if $M=\bigcap_{P \in \Lambda} M_{P}$, where $\Lambda \subseteq \operatorname{Spec}(R)$, and each localization $M_{P}$ of $M$ at $P \in \Lambda$ is a valuation module. 
Theorem 2.7. Let $M$ be a faithful multiplication module over an integral domain $R$. Suppose that for each nonzero submodule $N$ of $M, N_{v}$ is of finite type. Then the following are equivalent.

(1) Every nonzero submodule of $M$ is v-invertible.

(2) $M$ is a v-Prüfer module.

(3) $M$ is a t-Prüfer module.

(4) $M$ is an essential module.

Proof. (4) $\Rightarrow(1) \Rightarrow(2)$ are trivial.

$(2) \Rightarrow(3)$ This follows from [16, Theorem 2.5] and Lemma 2.5

$(3) \Rightarrow(4)$ Let $I$ be a finitely generated ideal of $R$. Then $I M$ is a finitely generated submodule. By hypothesis, $I M$ is $t$-invertible. Then $I$ is $t$-invertible, and so $R$ is a $t$-Prüfer domain. Then $R$ is an essential domain. Hence $M$ is an essential module [16, Theorem 2.5].

Recall from [16] that $M$ is called a Mori module if $M$ satisfies the ascending chain condition on $v$-submodules of $M$. If $M$ is a faithful multiplication and Mori module, then for each nonzero submodule $N$ of $M, N_{v}$ is of finite type by [16, Theorem 2.7].

Corollary 2.8. Let $R$ be an integral domain and $M$ a faithful multiplication and Mori module. Then the following are equivalent.

(1) Every nonzero submodule of $M$ is v-invertible.

(2) $M$ is a Krull module.

(3) $M$ is a v-Prüfer module.

(4) $M$ is a t-Prüfer module.

(5) $M$ is an essential module.

\section{3. $t$-invertible Submodules}

In this section we give several properties of a $t$-invertible submodule of a faithful multiplication module.

Proposition 3.1. Let $M$ be a faithful multiplication module over an integral domain $R$. If every nonzero prime submodule of $M$ contains a t-invertible prime submodule, then there exists a nonempty collection $\Lambda$ of minimal prime ideals of $R$ such that $M=\bigcap_{P \in \Lambda} M_{P}$ and each $M_{P}, P \in \Lambda$, is a CSM $R_{P}$-module.

Proof. Let $I$ be any nonzero prime ideal of $R$. By hypothesis, there exists a $t$-invertible prime submodule $N$ of $M$ such that $N \subseteq I M$. Then $I$ contains a $t$ invertible prime ideal $\left(N:_{R} M\right)$ of $R$. By [15, Lemma 3.3], there exists a nonempty collection $\Lambda$ of minimal prime ideals of $R$ such that $R=\bigcap_{P \in \Lambda} R_{P}$ and each $R_{P}$, 
$P \in \Lambda$, is a PID. By [5, Proposition 2.2], $M_{P}$ is a CSM $R_{P}$-module. Now we show that $M=\bigcap_{P \in \Lambda} M_{P}$. Clearly $M \subseteq \bigcap_{P \in \Lambda} M_{P}$. Let $0 \neq x \in \bigcap_{P \in \Lambda} M_{P}$. By [16, Lemma 2.4], $\left(M:_{R} R x\right) \not \subset P$ for each $P \in \Lambda$. Since $\left(M:_{R} R x\right)$ is a $t$-ideal and every proper $t$-ideal is contained in a maximal $t$-ideal of $R,\left(M:_{R} R x\right)=R$. Hence $x \in M$.

Let $M$ be a faithful multiplication module over an integral domain $R$. Then $M$ is finitely generated and torsion-free. Also, for every prime ideal $P$ of $R, M_{P} \cong R_{P}$ as an $R_{P}$-module. It follows that $N_{P} \cong\left(N:_{R} M\right)_{P}$, where $N$ is a submodule of $M$ [3].

Lemma 3.2. Let $M$ be a faithful multiplication module over an integral domain $R$. Suppose that $N$ is a finitely presented submodule of $M$. Then

(1) $\left(N:_{R} M\right)_{P} \cong\left(N_{P}: M_{P}\right)$ for every prime ideal $P$ of $R$.

(2) $\left(N:_{R} M\right)_{P}$ is a principal ideal of $R_{P}$ if and only if $N_{P}$ is a cyclic $R_{P}$-module for every prime ideal $P$ of $R$.

Proof. (1) Since $M$ and $N$ are torsion-fee, we have $\left(N:_{R} M\right)_{P} \cong \operatorname{Hom}(M, N)_{P}$. Also, $\operatorname{Hom}(M, N)_{P} \cong \operatorname{Hom}\left(M_{P}, N_{P}\right)$ since $N$ is finitely presented. Therefore $\left(N:_{R}\right.$ $M)_{P} \cong\left(N_{P}: M_{P}\right)$. (2) follows from (1).

Recall that an integral domain $R$ is called a coherent domain if every finitely generated ideal of $\mathrm{R}$ is finitely presented. Then it is well known that $R$ is a coherent domain if and only if every finitely generated torsion-free $R$-module is finitely presented. Therefore, if $R$ is a coherent domain and $N$ is finitely generated, then Lemma 3.2 holds.

The following result generalizes [17, Lemma 1.5 and Corollary 1.6].

Proposition 3.3. Let $M$ be a faithful multiplication module over an integral domain $R, N$ a finitely generated submodule of $M$. If $N$ is finitely presented, then $N$ is tinvertible if and only $N_{P}$ is a cyclic $R_{P}$-module for every maximal t-ideal $P$ of $R$.

Proof. Since $N$ is a finitely generated submodule of $M$, we have $\left(N:_{R} M\right)$ is a finitely generated ideal of $R$. If $N$ is $t$-invertible, then by [16, Proposition 2.2], $\left(N:_{R} M\right)$ is a $t$-invertible ideal of $R$. By [17, Lemma 1.5], $\left(N:_{R} M\right)_{P}$ is principal for every maximal $t$-ideal $P$ of $R$. Hence $N_{P}$ is a cyclic $R_{P}$-module. The proof of the converse is similar.

Corollary 3.4. Let $M$ be a faithful multiplication module over an integral domain $R, N$ a v-submodule of finite type. If $N$ is finitely presented, then $N$ is $t$-invertible if and only $N_{P}$ is a cyclic $R_{P}$-module for every maximal $t$-ideal $P$ of $R$.

Proof. By Lemma 2.1, $N$ is a $v$-submodule of finite type if and only if $\left(N:_{R} M\right)$ is a $v$-ideal of finite type. Then by [17, Corollary 1.6], $N$ is $t$-invertible, if and only if $\left(N:_{R} M\right)_{P}$ is principal for every maximal $t$-ideal $P$ of $R$, if and only if $N_{P}$ is a cyclic $R_{P}$-module. 
Let $M$ be a faithful multiplication module over an integral domain $R$. If $N$ is a $t$-invertible prime $t$-submodule of $M$, then $N$ is a maximal $t$-submodule of $M$.

The following result generalizes [17, Theorem 1 and Corollary 2].

Proposition 3.5. Let $M$ be a faithful multiplication module over an integral domain $R$ and let $N$ be a nonzero submodule of $M$. If every prime $t$-submodule minimal over $N$ is of finite type, then there are only finitely many $t$-submodules minimal over $N$.

Proof. Let $I$ be a prime $t$-ideal minimal over $\left(N:_{R} M\right)$. Then $I M$ is a prime $t$ submodule minimal over $N$. By hypothesis, $I M$ is of finite type, and so by Lemma $2.1, I$ is of finite type. Then there are only finitely many prime $t$-ideals $\left\{P_{1}, \cdots, P_{n}\right\}$ minimal over $\left(N:_{R} M\right)$ by [11, Theorem 1]. Hence $P_{1} M, \cdots, P_{n} M$ are only finitely many prime $t$-submodules minimal over $N$.

Corollary 3.6. Let $M$ be a faithful multiplication module over an integral domain $R$ and let $N$ be a nonzero submodule of $M$. If every prime $t$-submodule minimal over $N$ is t-invertible, then $N$ is contained in only finitely many minimal prime t-submodules.

Proof. This follows from the fact that every $t$-invertible $t$-submodule is of finite type.

Lemma 3.7. Let $M$ be a faithful multiplication module over an integral domain $R$, $J$ a finitely generated ideal of $R$ and $N$ a proper $v$-submodule of $M$. If $(J N)_{v}=$ $J_{v} N_{v}$, then $(I N)_{t}=I_{t} N_{t}$ for every ideal I of $R$.

Proof. Recall that $(I N)_{t}=\bigcup\left\{L_{v} \mid L\right.$ is a finitely generated submodule of $M$ contained in $I N\}$. For each $L$ in the definition, $L \subseteq J N$ for some finitely generated ideal $J \subseteq I$. Then $L_{v} \subseteq(J N)_{v}=J_{v} N_{v} \subseteq I_{t} N_{t}$. Thus $(I N)_{t} \subseteq I_{t} N_{t}$. The reverse inclusion is trivial.

Corollary 3.8. Let $R$ be a Dedekind domain and let $M$ be a faithful multiplication $R$-module, $I$ an ideal of $R$, and $N$ a proper submodule of $M$. Then $(I N)_{t}=I_{t} N_{t}$.

Following [14], a $T V$-domain is a domain in which every $t$-ideal is a $v$-ideal. Noetherian domains and Krull domains are TV-domains, cf. [14, p. 291].

Corollary 3.9. Let $R$ be a $T V$-domain and let $M$ be a faithful multiplication $R$ module, $J$ a finitely generated ideal of $R$, and $N$ a proper submodule of $M$. If $(J N)_{v}=J_{v} N_{v}$, then $(I N)_{t}=I_{t} N_{t}$ for every ideal $I$ of $R$.

Corollary 3.10. Let $M$ be a faithful multiplication module over an integral domain $R, J$ a finitely generated ideal of $R$ and $N$ a proper $v$-submodule of $M$. If $(J N)_{v}=$ $J_{v} N_{v}$, then $I N$ is a t-submodule of $M$ for every $t$-ideal $I$ of $R$.

The following result generalizes [17, Corollary 4 and Corollary 5]. 
Theorem 3.11. Let $M$ be a faithful multiplication module over an integral domain $R, J$ a finitely generated ideal of $R$ and $N$ a proper $v$-submodule of $M$. If $(J N)_{v}=$ $J_{v} N_{v}$, then the following are equivalent.

(1) $N=\left(P_{1}^{a_{1}} \cdots P_{n}^{a_{n}}\right)_{t} N^{*}$, where $P_{1}, \cdots, P_{n}$ are $t$-invertible prime $t$-ideals of $R$ and $N^{*}$ is a t-invertible prime $t$-submodule of $M$.

(2) Every prime t-submodule minimal over $N$ is t-invertible.

(3) Every prime t-submodule containing $N$ is t-invertible.

(4) There is a finite set of $t$-invertible prime $t$-ideals $\left\{P_{1}, \cdots, P_{n}\right\}$ and a $t$ invertible prime t-submodule $N^{*}$ of $M$ such that for every $t$-submodule $L$ containing $N, L=\left(P_{1}^{a_{1}} \cdots P_{n}^{a_{n}}\right)_{t} N^{*}$.

(5) Every t-submodule containing $N$ is t-invertible.

Moreover, in the case where (1) - (5) hold, every $t$-submodule $L$ containing $N$ is a $v$-submodule of finite type and the set of t-submodules containing $N$ is finite.

Proof. Obviously $(2) \Rightarrow(3)$ and $(4) \Rightarrow(1)$. $[16$, Lemma 2.1] shows that $(4) \Rightarrow(5)$. Clearly $(5) \Rightarrow(3)$.

Now we show that $(1) \Rightarrow(2)$. Suppose that $N=\left(P_{1}^{a_{1}} \cdots P_{n}^{a_{n}}\right)_{t} N^{*}$, where $P_{1}, \cdots, P_{n}$ are $t$-invertible prime $t$-ideals of $R$ and $N^{*}$ is a $t$-invertible

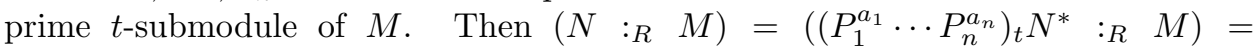
$\left(P_{1}^{a_{1}} \cdots P_{n}^{a_{n}}\right)_{t}\left(N^{*}:_{R} M\right)=\left(P_{1}^{a_{1}} \cdots P_{n}^{a_{n}}\left(N^{*}: M\right)\right)_{t}$ for each nonnegative inter $a_{i}$, since $\left(N:_{R} M\right)$ and $\left(N^{*}:_{R} M\right)$ are $t$-ideals of $R$. By [11, Corollary 4], every prime $t$-ideal minimal over $\left(N:_{R} M\right)$ is $t$-invertible. Hence every prime $t$-submodule minimal over $N$ is $t$-invertible.

$(3) \Rightarrow(4)$ Suppose that every prime $t$-submodules containing $N$ is $t$-invertible. By Corollary 3.6, the set of prime $t$-submodules over $N$ is finite, say $\left\{L_{1}, \cdots, L_{n}\right\}$. Then $\left\{Q_{1}, \cdots, Q_{n}\right\}$ is the set of prime $t$-ideals minimal over $\left(N:_{R} M\right)$, where $Q_{i}=$ $\left(L_{i}:_{R} M\right)$. Since each $L_{i}$ is $t$-invertible, each $Q_{i}=\left(L_{i}: M\right)$ is $t$-invertible by [16, Proposition 2.2]. Then there is a finite set of $t$-invertible prime $t$-ideals $\left\{P_{1}, \cdots, P_{n}\right\}$ such that every $t$-ideal containing $\left(N:_{R} M\right)$ is a $t$-product of powers of the $P_{i}$ 's. If $L$ is a $t$-submodule containing $N$, then $\left(L:_{R} M\right)=\left(P_{1}^{a_{1}} \cdots P_{n}^{a_{n}}\right)_{t}$ for each nonnegative inter $a_{i}$. Since $L=\left(L:_{R} M\right) M$, we have that $L_{t}=\left(P_{1}^{a_{1}} \cdots P_{n}^{a_{n}} M\right)_{t}=$ $\left(P_{1}^{a_{1}} \cdots P_{n}^{a_{n}-1} P_{n} M\right)_{t}=\left(I N^{*}\right)_{t}$, where $I=\left(P_{1}^{a_{1}} \cdots P_{n}^{a_{n}-1}\right)_{t}$ and $N^{*}=P_{n} M$. By Lemma 2.7, $L_{t}=\left(I N^{*}\right)_{t}=I_{t}\left(N^{*}\right)_{t}$. Hence $L=L_{t} M=I_{t}\left(N^{*}\right)_{t} M=I_{t} N^{*}=$ $\left(P_{1}^{a_{1}} \cdots P_{n}^{a_{n}-1}\right)_{t} N^{*}$.

Suppose that (1) - (5) hold for $N$ and let $L$ be a $t$-submodules containing $N$. Then there is a finite set of $t$-invertible prime $t$-ideals $\left\{Q_{1}, \cdots, Q_{n}\right\}$ and a $t$ invertible prime $t$-submodule $N^{*}$ of $M$ such that $L=\left(Q_{1}^{a_{1}} \cdots Q_{n}^{a_{n}}\right)_{t} N^{*}$. Hence $L$ itself is $t$-invertible by [16, Lemma 2.1]. Then $\left(L:_{R} M\right)$ is a $t$-invertible $t$-ideal, and so $\left(L:_{R} M\right)$ is a $v$-ideal. Therefore $L=\left(L:_{R} M\right) M$ is a $v$-submodule. Finally, since the set of $t$-submodules containing $N$ is $\left\{\left(P_{1}^{b_{1}} \cdots P_{n}^{b_{n}}\right)_{t} N^{*} \mid 0 \leq b_{i} \leq a_{i}\right\}$, where $N=\left(P_{1}^{a_{1}} \cdots P_{n}^{a_{n}}\right)_{t} N^{*}$, the set of $t$-submodules containing $N$ is finite. 
Proposition 3.12. Let $M$ be a faithful multiplication module over an integral domain $R, N$ a nonzero submodule of $M$. If $N=P_{1} \cdots P_{n} N^{*}$, where each $P_{i}$ is an invertible prime ideal of $R$ and $N^{*}$ is an invertible prime submodule of $M$, then every $v$-submodule containing $N$ is invertible.

Proof. Let $L$ be a $v$-submodule containing $N$. Then $\left(L:_{R} M\right)$ is a $v$-ideal containing $\left(N:_{R} M\right)$. Since $\left(N:_{R} M\right)=P_{1} \cdots P_{n}\left(N^{*}:_{R} M\right)$ and $\left(N^{*}:_{R} M\right)$ is invertible, $\left(L:_{R} M\right)$ is invertible by [17, Corollary 5]. Hence $L$ is invertible.

\section{References}

[1] F. H. Al-Alwan and A. G. Naoum, Dedekind modules, Comm. Algebra, 24(1996), 397-421.

[2] F. H. Al-Alwan and A. G. Naoum, Dense submodules of multiplication modules, Comm. Algebra, 24(1996), 413-424.

[3] M. Ali, Invertiblity of multiplication modules, New Zealand J. Math., 35(2006), 17-29.

[4] M. Ali, Some remarks on generalized GCD domains, Comm. Algebra, 36(2008), 142164.

[5] M. Ali, Invertiblity of multiplication modules II, New Zealand J. Math., 39(2009), 45-64.

[6] M. Ali and D. J. Smith, Some remarks on multiplication and projective modules, Comm. Algebra, 32(2004), 3897-3909.

[7] M. Alkan, B Saraç, and Y. Tiraş, Dedekind modules, Comm. Algebra, 33(2005), 1617-1626.

[8] M. Alkan and Y. Tiras, Prime modules and submodules, Comm. Algebra, 31(2003), 5253-5261.

[9] M. Alkan and Y. Tiraş, On Invertible and dense submodules, Comm. Algebra, 32(2004), 3911-3919.

[10] Y. Al-Shaniafi and D. D Anderson, Multiplication modules and the ideal $\Theta(M)$, Comm. Algebra, 30(2002), 3383-3390.

[11] D. D. Anderson, On t-invertibility IV, Factorization in integral domains (Iowa City, IA, 1996), 221-225, Lecture Notes in Pure and Appl. Math., 189, Dekker, New York, 1997.

[12] Z. A. El-Bast and P. F. Smith, Multiplication modules, Comm. Algebra, 16(1988), 755-739.

[13] V. Erdogdu, Multiplication modules which are distributive, J. Pure Appl. Algebra, 54(1988), 209-213.

[14] E. Houston and M. Zafrullah, Integral domains in which each t-ideal is divisorial, Mich. Math. J. 35(1988), 291-300.

[15] B. G. Kang, On the converse of a well-known fact about Krull domains, J. Algebra, 124(1989), 284-299. 
[16] H. Kim and M. O. Kim , Krull modules, Algebra Colloq., 20(2013), 464-474.

[17] S. Malik, J. L. Mott, and M. Zafrullah, On t-invertibility, Comm. Algebra, 16(1988), 149-170.

[18] J. L. Mott and M. Zafrullah, On Krull domains, Arch. Math., 56(1991), 559-568.

[19] P. F. Smith, Some remarks on multiplication modules, Arch. Math., 50(1988), 223235.

[20] M. Zafrullah, Ascending chain condition and star operations, Comm. Algebra, 17(1989), 1523-1533. 